\title{
Differential tunnel transparency of a rectangular heterostructural barrier for the terahertz frequency range
}

\author{
Z. S. Gribnikov ${ }^{\text {a) }}$ \\ Institute for Quantum Sciences, Michigan State University, East Lansing, Michigan 48824 \\ and Department of Electrical Engineering and Computer Science (EECS), University of Michigan, \\ Ann Arbor, Michigan 48109 \\ G. I. Haddad \\ Department of Electrical Engineering and Computer Science (EECS), University of Michigan, \\ Ann Arbor, Michigan 48109
}

(Received 6 August 2004; accepted 14 February 2005; published online 18 April 2005)

\begin{abstract}
Electron wave tunneling through a rectangular heterostructural emitter barrier is considered in the case of a homogeneous high-frequency (hf) alternating electric field directed normal to the barrier interfaces. This hf field leads not only to the well-known increase in a stationary tunnel current through the emitter barrier, which is proportional to $E_{B}^{2}$ (where $E_{B}$ is the electric-field amplitude) but also to a linear $\left(\sim E_{B}\right)$ increase in an alternating current (ac) through this barrier with the same frequency $\omega$ as the electric-field frequency. The ac is a sharp function of $\omega$, which grows significantly with an increase in $\omega$ (typically in the terahertz range). In a certain intermediate current and frequency region, the above-mentioned increase in the ac is the dominating effect of the alternating field. Such an effect can be used to optimize tunnel barrier emitters for ballistic transit-time terahertz-range oscillators. (C) 2005 American Institute of Physics. [DOI: 10.1063/1.1886277]
\end{abstract}

\section{INTRODUCTION}

In a previous article, ${ }^{1}$ we considered several models relating to a time-dependent electron tunneling through nonstationary tunnel emitter barriers. As is known, ${ }^{2-5}$ the quasistatic approach based on the use of the static tunneling equations becomes incorrect if a characteristic frequency tends to the terahertz range. In particular, this range can be realistic in the case of quasiballistic and ballistic transit-time diode oscillators suggested and considered in Refs. 6-9 [the so-called ballistic tunnel emission transit-time (TUNNETT) diodes]. In such diodes, the above-mentioned nonstationary tunnel barriers should serve as high-frequency electron emitters. In the two examples considered previously, ${ }^{1}$ in (1) the rectangular barrier with a time-dependent height and in (2) the rectangular barrier with a time-dependent $\delta$-function perturbation localized in an arbitrary position inside the barrier, a substantial increase in high-frequency current takes place when a frequency, $\omega=2 \pi f$, exceeds the inverse time for tunneling $^{2}$ through a static rectangular barrier. Both these examples (despite their clarity) are hard to attain experimentally. Here, we consider one more model with many more accessible experimental implementations. We consider the same rectangular barrier $B$ (see Fig. 1) enclosed between homogeneous regions 1 and 2 . An electron current with en$\operatorname{ergy} \varepsilon$ (and without transverse momentum, $p_{\perp} \cong 0$ ), which is incident from the left region (region 1), is partially reflected and partially transmitted to the right region (region 2) where its kinetic energy is supposedly higher by a value $\varepsilon_{12}^{(0)}$. We assume that the alternating high-frequency voltage

${ }^{a)}$ Electronic mail: gribnikov@pa.msu.edu

$$
U=E_{B} w \cos \omega t
$$

biases the barrier and induces a homogeneous electric field $E=E_{B} \cos \omega t$ inside. The barrier height is modulated by the value $e U(x, t)=e E_{B} x \cos \omega t$. Since the conduction-band discontinuity in the interface between the barrier and region 2 does not depend on the field $E(t)$, the above-introduced increase in kinetic energy in region 2 (see Fig. 1) is also time modulated:

$$
\varepsilon_{12}(t)=\varepsilon_{12}^{(0)}-e E_{B} w \cos \omega t
$$

In reality, an electric field in region 2 could not be abruptly eliminated. A certain field should exist there: it is caused by the continuity of a normal component of the electric induction vector $D_{x}=\kappa_{D} E_{x}$ in the interface of the barrier/region 2 where $\kappa_{D}$ is a dielectric constant. The existence of such a field is a substantial element of a theory of the abovementioned transit-time diodes (including the ballistic TUN-

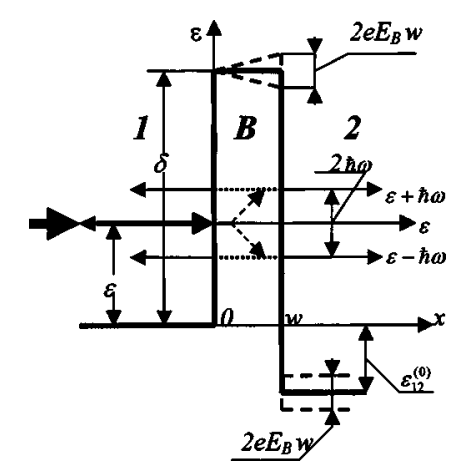

FIG. 1. Rectangular heterostructural barrier with a homogeneous alternating electric field. 
NETT diodes ${ }^{7-9}$ ). Just an interaction of this field with an inertial emitted electron current in region 2 is responsible for an oscillatory regime. But the tunnel emission through a rectangular barrier as such depends on this field in region 2 weakly. This fact allows us to neglect it in our consideration here. The above-mentioned region 2 is nothing but the socalled transit space $^{7-9}$ (or the drift region) in our transit-time diode. This space is completely depleted in the operating conditions. There are not any electrons in this space excluding the above-considered ballistic electrons emitted from the left. Therefore, there is not any opposite tunnel current through the tunnel emitter barrier from the right to left.

As in the previous article, ${ }^{1}$ we consider here the simplest quantum-mechanical problem relating to transmission and reflection of a single-electron wave. In reality, we deal with a multielectron current, in which electrons with different values of energy $\varepsilon$ and transverse momentums $p_{\perp}$ participate. But in the most interesting case of a small tunnel barrier transparency (at $\varepsilon=\varepsilon_{F}$, where $\varepsilon_{F}$ is a Fermi energy in the depth of region 1) and a low temperature of an electron gas in the same region 1 , a tunnel current is determined by electrons with small values of $p_{\perp}$ and with values of $\varepsilon$ close to $\varepsilon_{F}$. Therefore, the simplest proposed consideration gives a credible frequency behavior of a time-dependent tunnel electron current. Of course, such a consideration can serve only as a preliminary introduction for a detailed theory.

\section{EQUATIONS AND SOLUTIONS}

\section{A. Equations}

We need to solve the Schrödinger equation in both regions 1 and 2 and in the barrier $B$, respectively,

$$
\begin{aligned}
& i \hbar \frac{\partial \Psi_{1}}{\partial t}=-\frac{\hbar^{2}}{2 m_{1}} \frac{\partial^{2} \Psi_{1}}{\partial x^{2}}, \\
& i \hbar \frac{\partial \Psi_{2}}{\partial t}=-\frac{\hbar^{2}}{2 m_{2}} \frac{\partial^{2} \Psi_{2}}{\partial x^{2}}-\left[\varepsilon_{12}^{(0)}-e E_{B} w \cos \omega t\right] \Psi_{2}, \\
& i \hbar \frac{\partial \Psi}{\partial t}=-\frac{\hbar^{2}}{2 m} \frac{\partial^{2} \Psi}{\partial x^{2}}+\left(\delta+e E_{B} x \cos \omega t\right) \Psi,
\end{aligned}
$$

where $\Psi_{1}(x, t), \Psi_{2}(x, t)$, and $\Psi(x, t)$ are wave functions in the above-listed areas, and $m_{1}, m_{2}$, and $m$ are electron effective masses in the same areas. It is assumed that an electron wave with energy $\varepsilon$ incident from the left generates, as a result of its interaction with the time-dependent barrier $B$, a transmitted wave in region 2 , and a reflected wave in region 1 with the same energy $\varepsilon$, and also "transmitted" and "reflected" waves with energies $\varepsilon \pm \hbar \omega, \varepsilon \pm 2 \hbar \omega, \varepsilon \pm 3 \hbar \omega$, etc. The first two pairs are shown in Fig. 1. The spectrum of these waves is restricted from the bottom by the real bottoms of the conduction bands in regions 1 and 2. The quantities of additional waves, which really take part in a tunnel emission, depend on the value of the nonstationary barrier perturbation. In our specific case, such a defining value is the amplitude $e E_{B} w$, which should be compared not only with the energy height of the effective barrier $\delta-\varepsilon$, and not only with energies $\varepsilon$ and $\varepsilon+\varepsilon_{12}^{(0)}$, but also with energy $\hbar \omega$. [Let us note that the frequency $\omega$ is present in Eqs. (4) and (5) only in the form cos $\omega t$.] In the simplest case of a small amplitude $e E_{B} w$, we can consider only the wave triad with energies $\varepsilon$ and $\varepsilon \pm \hbar \omega$.

\section{B. Static case}

Let us consider the solutions of Eqs. (3)-(5) in the static case when $\omega=0$ and $E=E_{B}$. In Eq. (4) there is the only replacement $\varepsilon_{12}^{(0)} \rightarrow \varepsilon_{12}^{(0)}-e E w$. Equation (5) can be rewritten in the form

$$
\frac{d^{2} \Phi}{d \xi^{2}}-\left(1+\xi_{0}^{-1} \xi\right) \Phi=0,
$$

where $\Psi(x, t)=\Phi(\xi) \exp (-i \varepsilon t / \hbar), \quad \xi=g x, \quad g=\sqrt{\mu(\delta-\varepsilon)}, \quad \mu$ $=2 m / \hbar^{2}$, and $\xi_{0}=g(\delta-\varepsilon) / e E$. The accurate solution of Eq. (6) can be presented in the form

$$
\Phi(\xi)=C_{1} \operatorname{Ai}\left(\xi_{0}^{2 / 3}+\xi_{0}^{1 / 3} \xi\right)+S_{1} \operatorname{Bi}\left(\xi_{0}^{2 / 3}+\xi_{0}^{1 / 3} \xi\right),
$$

where $\operatorname{Ai}(z)$ and $\operatorname{Bi}(z)$ are the Airy functions. ${ }^{10}$ We consider only the case of comparatively small fields when

$$
\xi_{0} \gg 1
$$

This means that $\xi_{0}^{2 / 3} \gg 1$ also. So arguments of the Airy functions in Eq. (7) are large and we can use asymptotic expansions of these functions: ${ }^{10}$

$$
\begin{aligned}
\Phi(\xi) \cong & {\left[1+\left(\xi / \xi_{0}\right)\right]^{-1 / 4}\left\{C_{2} \exp \left[-(2 / 3) \xi_{0}\left(1+\xi_{0}^{1} \xi\right)^{3 / 2}\right]\right.} \\
& \left.+S_{2} \exp \left[(2 / 3) \xi_{0}\left(1+\xi_{0}^{-1} \xi\right)^{3 / 2}\right]\right\} \\
\cong & {\left[1-\left(\xi / 4 \xi_{0}\right)\right]\left\{C \exp \left[-\xi-(1 / 4) \xi_{0}^{-1} \xi^{2}\right]\right.} \\
& \left.+S \exp \left[\xi+(1 / 4) \xi_{0}^{-1} \xi^{2}\right]\right\} \\
= & (1-\gamma x)\{C \exp [-g x(1+\gamma x)] \\
& +S \exp [g x(1+\gamma x)]\},
\end{aligned}
$$

where $\gamma=e E / 4(\delta-\varepsilon)$.

In the middle lines of Eq. (9), we have used the additional assumption $g w \ll \xi_{0}$ or $e E w \ll \delta-\varepsilon$. The solution $\Phi(\gamma x)$ in the barrier should be combined with analogous static solutions of Eqs. (3) and (4) in regions 1 and 2:

$$
\Phi_{1}=\exp \left(i k_{1} x\right)+B \exp \left(-i k_{1} x\right)
$$

and

$$
\Phi_{2}=F \exp \left[i k_{2}(E) x\right],
$$

where $k_{1}=\left(\mu_{1} \varepsilon\right)^{1 / 2}, k_{2}(E)=\left\{\mu_{2}\left[\varepsilon+\varepsilon_{12}^{(0)}-e E w\right]\right\}^{1 / 2}$, and $\mu_{1,2}$ $=2 m_{1,2} / \hbar^{2}$.

We equate in the boundaries of the barrier $x=0$ and $x=w$ functions $\Phi_{1}$ to $\Phi$ and $\Phi$ to $\Phi_{2}$, respectively, and also, respectively, $\left(1 / \mu_{1}\right)\left(d \Phi_{1} / d x\right)$ to $(1 / \mu)(d \Phi / d x)$ and $(1 / \mu)(d \Phi / d x)$ to $\left(1 / \mu_{2}\right)\left(d \Phi_{2} / d x\right)$. As a result, we obtain the system of equations 
$1+B-C-S=0, \quad i \lambda_{0}(1-B)+(C-S)=-\gamma(1+B) / g$,

$F-C \exp \left(-g^{\prime} w\right)-S \exp \left(g^{\prime} w\right)=-\gamma F w$

$i \lambda F+C \exp \left(-g^{\prime} w\right)-S \exp \left(g^{\prime} w\right)=\gamma F\left[i \lambda\left(w+w_{k}\right)-g^{-1}\right]$,

where $g^{\prime}=g(1+\gamma w), \lambda=k_{2}(0) \mu / g \mu_{2}, \lambda_{0}=k_{1} \mu / g \mu_{1}$, and $w_{k}$ $=2 w(\delta-\varepsilon) /\left[\varepsilon+\varepsilon_{12}^{(0)}\right]$. The electric-field corrections introduced by the right-hand sides of Eqs. (12a)-(12c) lead to corrections in the tunnel transparency having an order of accuracy of $\sim e E w / 4(\delta-\varepsilon)$, or $e E / 4(\delta-\varepsilon) g$, or $e E w / 2[\varepsilon$ $\left.+\varepsilon_{12}^{(0)}\right]$. We assume all these values are small and we can neglect these corrections. The main electric-field correction is introduced as a result of replacement of $g$ by $g^{\prime}$ in the exponents in Eqs. (12b) and (12c). Taking into account only this correction, we obtain from Eqs. (12a)-(12c)

$$
F=\frac{2(1+\nu) \exp \left(-g^{\prime} w\right)}{(1+i \chi)\left[1+\nu \exp \left(-2 g^{\prime} w\right)\right]},
$$

where $\nu=(1+i \lambda) /(1-i \lambda)$, and $\chi=\left[1-\nu \exp \left(-2 g^{\prime} w\right)\right] / \lambda_{0}[1$ $\left.+\nu \exp \left(-2 g^{\prime} w\right)\right]$. In the case of the thick barrier when $g w$ $\gg 1$, we can neglect the contribution of components of $\left.\sim \nu \exp \left(-2 g^{\prime} w\right)\right]$. Then we obtain for the tunneling current

$$
\begin{aligned}
j(E) & \cong\left(\hbar k_{2} / m_{2}\right)|F|^{2} \\
& \cong j(0) \exp \left[-2\left(g^{\prime}-g\right) w\right] \\
& =j(0) \exp \{-g w[e E w / 2(\delta-\varepsilon)]\},
\end{aligned}
$$

with $j(0) \cong\left[16 \lambda_{0}^{2} \hbar k_{2} / m_{2}\left(1+\lambda^{2}\right)\left(1+\lambda_{0}^{2}\right)\right] \exp (-2 g w)$. If $g w$ $\leqslant 1$, the correction introduced by the exponential multiplayer on the right-hand side of Eq. (14) is as small as the other corrections neglected above. But in the case of thick lowtransparency barriers when $g w \gg 1$, this correction is $g w$ or even $(g w)^{2}$ times larger and should be taken into account. If $g w$ is so large that $g w[e E w / 2(\delta-\varepsilon)]>1$, we obtain an exponential decrease in $j(E)$.

Let us assume now that in Eq. (14) $E=E_{0}+E_{B} \cos \omega t$ but frequency $\omega$ is so low that the static formula (14) is valid. If

$$
g w\left[e E_{B} w / 2(\delta-\varepsilon)\right] \ll 1,
$$

we have

$$
j(E) \cong j\left(E_{0}\right)\left\{1-\left[(g w) e E_{B} w / 2(\delta-\varepsilon)\right] \cos \omega t\right\} .
$$

The second component in the curly brackets in Eq. (16) is a quasistatic alternating current induced by the field component $E_{B} \cos \omega t$. If condition (15) is invalid, an expansion of $\exp \left\{-g w\left[e E_{B} w / 2(\delta-\varepsilon)\right] \cos \omega t\right\}$ into a series contains numerous harmonics of the basic frequency $\omega$.

\section{Substantially high-frequency case}

Turning to the substantially nonstationary problem, we assume that amplitude $E_{B}$ is sufficiently small and allows us to take into account only three values of electron energy, $\varepsilon$ $=\hbar \Omega$ and $\varepsilon \pm \hbar \omega=\hbar(\Omega \pm \omega)$, neglecting all the others. The electron components with energy $\varepsilon=\hbar \Omega$ are excited directly by the basic wave with the amplitude 1 and, therefore, they are much more intensive than the satellite components induced by the small alternating electric field with the amplitude $E_{B}$. This fact allows us to neglect this alternating field in Eqs. (4) and (5) considering these satellites and to write the solution of Eq. (3) in the form

$$
\begin{aligned}
\Psi_{1}(x, t)= & \exp (-i \Omega t)\left[\exp \left(i k_{1} x\right)+B \exp \left(-i k_{1} x\right)\right. \\
& +B^{(+)} \exp \left(-i k_{1+} x+i \omega t\right) \\
& \left.+B^{(-)} \exp \left(-i k_{1-} x-i \omega t\right)\right]
\end{aligned}
$$

and the solution of Eq. (5) in the form

$$
\begin{aligned}
\Psi_{2}(x, t)= & \exp (-i \Omega t)\left\{F \exp \left[i k_{2} x-i\left(e E_{B} w / \hbar \omega\right) \sin \omega t\right]\right. \\
& \left.+F^{(+)} \exp \left(i k_{2+} x+i \omega t\right)+F^{(-)} \exp \left(i k_{2-} x-i \omega t\right)\right\} .
\end{aligned}
$$

We have new designations in Eqs. (17) and (18): $k_{1 \pm}^{2}$ $=\mu_{1} \hbar(\Omega \mp \omega)=\mu_{1}(\varepsilon \mp \hbar \omega), k_{2 \pm}^{2}=\mu_{2} \hbar\left(\Omega+\Omega_{12} \mp \omega\right)$, and $\Omega_{12}$ $=\varepsilon_{12}^{(0)} / \hbar$. The above-mentioned condition of the small value of the electric-field amplitude, which can be written as

$$
A=e E_{B} w / \hbar \omega \ll 1,
$$

allows us to rewrite Eq. (18) in the approximate form

$$
\begin{aligned}
\Psi_{2}(x, t) \cong & \exp (-i \Omega t)\left\{F \exp \left(i k_{2} x\right)+\left[F^{(+)} \exp \left(i k_{2+} x\right)\right.\right. \\
& \left.-\left(e E_{B} w / 2 \hbar \omega\right) F \exp \left(i k_{2} x\right)\right] \exp (i \omega t) \\
& +\left[F^{(-)} \exp \left(i k_{2-} x\right)\right. \\
& \left.\left.+\left(e E_{B} w / 2 \hbar \omega\right) F \exp \left(i k_{2} x\right)\right] \exp (-i \omega t)\right\} .
\end{aligned}
$$

At last, we obtain the function $\Psi(x, t)$ describing the decaying tunnel wave in the barrier $B$ and determined by Eq. (4), from which we can obtain

$$
\begin{aligned}
\Psi(x, t) \cong & e^{-i \Omega t}\left\{C e^{-g x}+S e^{g x}+\left[C^{(+)} e^{-g_{+} x}+S^{(+)} e^{g_{+} x}\right] e^{i \omega t}\right. \\
& +\left[C^{(-)} e^{-g_{-} x}+S^{(-)} e^{g_{-} x}\right] e^{-i \omega t} \\
& +\left[2 e E_{B} g / \mu(\hbar \omega)^{2}\right]\left(C e^{-g x}-S e^{g x}\right) \cos \omega t \\
& \left.-\left(i e E_{B} x / \hbar \omega\right)\left(C e^{-g x}+S e^{g x}\right) \sin \omega t\right\},
\end{aligned}
$$

where $g_{ \pm}=\sqrt{\mu(\delta-\varepsilon \pm \hbar \omega)}$. Solution (21) in the barrier is equivalent to solution (20) in region 2 [but in no way equivalent to the more general solution (18)]. It is valid if condition (19) is satisfied as well as the more intricate condition

$$
2 \beta=2 e E_{B} g / \mu(\hbar \omega)^{2} \leqslant 1 .
$$

The constant $B, C, S$, and $F$ in solutions (17), (20), and (21) are the same as in Eqs. (10), (11), and (9). They should be calculated from Eqs. (12a)-(12c) for $\gamma=0 \quad\left(E_{0}=0\right)$. The analogous boundary conditions, which allow us to calculate the constants $B^{( \pm)}, C^{( \pm)}, S^{( \pm)}$, and $F^{( \pm)}$, take into account the appearance of components that are proportional to $\exp ( \pm i \omega t)$ on the right sides of Eqs. (20) and (21). [In Eq. (21) we need to remember that $\cos \omega t=\left(e^{i \omega t}+e^{-i \omega t}\right) / 2$ and $\sin \omega t=-i\left(e^{i \omega t}\right.$ $\left.-e^{-i \omega t}\right) / 2$.] To calculate the alternating current in region 2 we need constants $F^{( \pm)}$, which are

$$
F^{( \pm)}=\Delta_{F}^{( \pm)} / \Delta^{( \pm)},
$$

where 


$$
\begin{aligned}
\Delta_{F}^{( \pm)}= & {\left[4 e E_{B} g / \mu(\hbar \omega)^{2}(1+i \chi)\left(1+\nu \bar{\alpha}^{2}\right)\right]\left[\left(g^{2}+g_{ \pm}^{2}\right)\right.} \\
& \times\left(1+\nu \bar{\alpha}^{2}\right) / 2 g g_{ \pm}-i \lambda_{0}^{ \pm}\left(1-\nu \bar{\alpha}^{2}\right)-\left(g^{2}+g_{ \pm}^{2}\right) \\
& \times(1+\nu) \bar{\alpha}\left(\cosh g_{ \pm} w-i \lambda_{0}^{ \pm} \sinh g_{ \pm} w\right) / 2 g g_{ \pm} \\
& \left.-(1-\nu) \bar{\alpha}\left(\sinh g_{ \pm} w-i \lambda_{0}^{ \pm} \cosh g_{ \pm} w\right)\right],
\end{aligned}
$$

$\bar{\alpha}=\exp (-g w)=\alpha^{-1}, \quad \lambda_{0}^{ \pm}=k_{1 \pm} \mu / g_{ \pm} \mu_{1}, \quad$ and $\lambda^{ \pm}=k_{2 \pm} \mu / g_{ \pm} \mu_{2}$. Knowing $F$ and $F^{( \pm)}$, we can find the desired current

$$
\begin{aligned}
j= & \left(\hbar k_{2} / m_{2}\right)|F|^{2}+\left(\hbar / 2 m_{2}\right)\left\{\left[\left(k_{2}+k_{2+}\right) F F^{(+)^{*}}\right.\right. \\
& \left.\left.+\left(k_{2}+k_{2-}\right) F^{*} F^{(-)}\right] e^{i\left(k_{2}-k_{2+}\right) x-i \omega t}+\text { c.c. }\right\}
\end{aligned}
$$

where c.c. means the complex-conjugate component.

To simplify our formula, we turn to the really interesting case of thick (low-transparency) barriers $(\bar{\alpha} \ll 1)$ and comparatively low frequencies $\left(\hbar \omega \ll \delta-\varepsilon, \varepsilon+\varepsilon_{12}\right)$. The latter allows us to approximately equate not only $k_{2 \pm} \cong k_{2}$ but also $g_{ \pm} \cong g$ everywhere excluding exponents. Using such simplifications, we obtain

$$
\Delta^{ \pm} \cong-\alpha^{ \pm}(1-i \lambda)\left(1-i \lambda_{0}\right), \quad \Delta_{F}^{ \pm} \cong-4 i \beta \lambda_{0}\left(1-\alpha^{ \pm} \bar{\alpha}\right),
$$

where $\alpha^{ \pm}=\exp \left(g_{ \pm} w\right)$. As a result of substitution of expressions (25) in Eq. (23), we obtain

$$
F^{( \pm)}=-\beta F\left(\bar{\alpha}^{ \pm} \alpha-1\right),
$$

with $\bar{\alpha}^{ \pm}=\exp \left(-g_{ \pm} w\right)$ and $F \cong-4 i \lambda_{0} \bar{\alpha} /\left(1-i \lambda_{0}\right)(1-i \lambda)$. At last, we obtain from Eq. (24)

$$
\begin{aligned}
j & \cong\left(\hbar k_{2} / m_{2}\right)|F|^{2}\left[1-2 \beta\left(\bar{\alpha}^{+} \alpha+\bar{\alpha}^{-} \alpha-2\right) \cos (\omega t-q x)\right] \\
& =j(0)[1-4 \beta(\cosh Q w-1) \cos (\omega-q x)],
\end{aligned}
$$

where $j(0)$ is the same as in Eq. (14), $Q w=(g w / 2)[\hbar \omega /(\delta$ $-\varepsilon)$, and $q=k_{2} \omega / 2\left(\Omega+\Omega_{12}\right)$. It is not difficult to verify that

$$
\beta=A / Q w
$$

where $A$ is introduced by Eq. (19). Therefore, Eq. (27) can be rewritten in the form

$$
j=j(0)\left[1-8 A \frac{\sinh ^{2}(Q w / 2)}{Q w} \cos (\omega t-q x)\right] .
$$

If the argument $Q w$ of the hyperbolic cosine in Eq. (27) is small in comparison to 1, we obtain from Eq. (27)

$$
\begin{aligned}
j & =j(0)\left[1-2 \beta Q^{2} w^{2} \cos (\omega t-q x)\right] \\
& =j(0)[1-2 A Q w \cos (\omega t-q x)] .
\end{aligned}
$$

This result (for $x=0$ ) coincides with Eq. (16) (for $E_{0}=0$ ). But for $Q w \geqslant 1$ when Eq. (30) is invalid and we need to use Eq. (27), there is a substantial difference: the high-frequency alternating current drastically increases with an increase in $\omega$.

\section{DISCUSSION}

Let us clarify the limits of the significant increase in the alternating current amplitude with an increase in frequency $\omega$ (for invariable other parameters of the problem including all the rectangular barrier parameters and the electric-field amplitude). In deriving Eq. (27) [or (29)] we have used the conditions of the small value of the electric-field amplitude in the form $A \ll 1$ [Eq. (19)] and $2 \beta=2 A / Q w \ll 1$ [Eq. (22)]. But these conditions do not really restrict any increase in the current because the latter is connected with an increase in $(\exp Q w) / Q w$ when frequency $\omega$ increases (at invariable $A$ $\ll 1)$.

We have also used the strong inequalities $F^{( \pm)} \ll F$ to derive Eq. (24) since in the latter all the components of the order of $\left|F^{( \pm)}\right|^{2}$ and $\left|F^{(+)^{*}} F^{(-)}\right|$are not taken into account. Such neglect requires the additional condition

$$
4 \beta(\cosh Q w-1)=4 A \sinh ^{2}(Q w / 2) / Q w \leqslant 1 .
$$

At last, we have considered only the triad of the electron waves with energies $\varepsilon$ and $\varepsilon \pm \hbar \omega$ but we have not taken into account the waves with energies $\varepsilon \pm 2 \hbar \omega$, etc. Such an assumption is valid for much weaker limitations than Eq. (31).

Let us write the expression for $j$ keeping all the triad components neglected before [in Eqs. (27) and (29)]:

$$
\begin{aligned}
j \cong & \left(\hbar k_{2} / m_{2}\right)\left(|F|^{2}+\left|F^{(+)}-A F\right|^{2}+\left|F^{(-)}+A F\right|^{2}\right. \\
& +\left\{\left[F F^{(+)^{*}}+F^{*} F^{(-)}\right] e^{i q x-i \omega t}+\text { c.c. }\right\} \\
& \left.+\left\{\left[F^{(+)^{*}}-A F^{*}\right]\left[F^{(-)}+A F\right] e^{2 i(q x-\omega t)}+\text { c.c. }\right\}\right) \\
\cong & j(0)\left\{1+4 A^{2}\left[\cosh Q w(\cosh Q w-1) /(Q w)^{2}\right.\right. \\
& +1 / 2-\sinh Q w / Q w]-4 A[(\cosh Q w-1) / Q w] \\
& \times \cos (\omega t-q x)-4 A^{2}\left[(\cosh Q w-1) /(Q w)^{2}+1 / 2\right. \\
& +\sinh Q w / Q w] \cos (2 \omega t-2 q x)\} .
\end{aligned}
$$

We can conclude from Eq. (32) that while $A \ll 1$ the last component in the outsize parentheses, which describes the $2 \omega$ harmonic of the alternating current, is always much smaller than each of the other components. We can also conclude that the alternating current (ac) component is always smaller than the dc component but there exists the frequency interval, in which this small ac component grows with an increase in $\omega$ much faster than the dc current. Such a behavior takes place just when inequality (31) is satisfied. But if instead of Eq. (31) the opposite strong inequality occurs:

$$
A \cosh Q w /(Q w) \gg 1
$$

(and $A \ll 1$ as before), we can observe a drastic increase in the dc component in comparison to the ac since the former is proportional to $[A \cosh Q w /(Q w)]^{2}$. We can hope that the optimal working regime for the high-frequency tunnel emitters can be realized if $(A / 2 Q w) \exp Q w \approx 1$ or $\left[e E_{B}(\delta\right.$ $-\varepsilon) / g \hbar \omega] \exp [\hbar \omega g w / 2(\delta-\varepsilon)] \approx 1$. Around such frequencies the portion of the alternating current with frequency $\omega$ is maximal in comparison to the full current, and it is possible to reach the maximal efficiency for the oscillators based on such emitters.

The analogous conclusion can be obtained on the basis of the simpler model considered before. ${ }^{1}$ In the case of the rectangular barrier with a time-dependent height $\varepsilon_{B}=\varepsilon_{0}$ $+\varepsilon^{(1)} \cos \omega t$, the formula, which is analogous to Eq. (32), appears as 


$$
\begin{aligned}
j \cong & j(0)\left[1+4 \alpha^{2} \cosh Q w(\cosh Q w-1)\right. \\
& -4 \alpha \sinh Q w \cos (\omega t-q x) \\
& \left.+4 \alpha^{2}(\cosh Q w-1) \cos 2(\omega t-q x)\right]
\end{aligned}
$$

where $\alpha=\varepsilon^{(1)} / 2 \omega$ is analogous to $A$ introduced by Eq. (19) and $Q$ is the frequency parameter of a rectangular tunnel barrier introduced in Eq. (27) here [and by Eq. (40) in Ref. 1].

\section{CONCLUSION}

We have derived formulas describing a one-electron tunnel current through a rectangular heterostructural emitter barrier containing a high-frequency homogeneous electric field with the amplitude $E_{B}$ inside. We have assumed that this amplitude is sufficiently small: $e E_{B} w \ll \delta-\varepsilon, \varepsilon, \hbar \omega$. In the thin-barrier case $(g w \leqslant 1)$, the quasistatic approach is always satisfactory. But in the thick-barrier case $(g w \gg 1)$, the known dynamic effects take place. These effects can be described with the help of two parameters: $A=e E_{B} w / 2 \hbar \omega \ll 1$ and $Q w=\hbar \omega g w / 2(\delta-\varepsilon)$. The latter can be varied in the arbitrary limits. For $Q w \ll 1$ the results of the quasistatic approach are suitable as before: neither a tunnel dc nor an ac amplitude depends on the frequency. For $Q w \geqslant 1$ the quasistatic approach becomes unsuitable: the ac amplitude experiences a fast growth with an increase in the frequency for the invariable amplitude $E_{B}$ and invariable rectangular barrier parameters. If $Q w \gg 1$ the relation of the ac amplitude to the tunnel $\mathrm{dc}$ is approximately equal to

$$
J_{\mathrm{ac}} / j_{\mathrm{dc}}=\frac{2 A \exp Q w /(Q w)}{1+[A \exp Q w /(Q w)]^{2}},
$$

that is for $A \exp Q w /(Q w) \cong 1$ the ac amplitude nears the dc. Implementation of such a regime in the ballistic transit-time oscillators could provide a noticeable advantage since it allows one to reach a comparatively high oscillatory power by using comparatively weak alternating fields in the emitter barrier and the depleted transit space (and to exceed an efficiency of the ballistic transit-time oscillators with the tunnel electron emission).

\section{ACKNOWLEDGMENTS}

The authors thank Dr. S. Tipton for the fruitful comments. One of the authors (Z.S.G.) thanks Mark Dykman for valuable discussions. This work was supported by the AFOSR through the MURI program F 4960-00-0328.

${ }^{1}$ Z. S. Gribnikov and G. I. Haddad, J. Appl. Phys. 96, 3831 (2004).

${ }^{2}$ M. Büttiker and R. Landauer, Phys. Rev. Lett. 49, 1739 (1982); Phys. Scr. 32, 429 (1985).

${ }^{3}$ B. I. Ivlev and V. I. Mel'nikov, Phys. Rev. Lett. 55, 1614 (1985).

${ }^{4}$ B. I. Ivlev and V. I. Mel'nikov, Zh. Eksp. Teor. Fiz. 90, 2208 (1986) [Sov. Phys. JETP 63, 1295 (1986)].

${ }^{5}$ R. Landauer and Th. Martin, Rev. Mod. Phys. 66, 217 (1994).

${ }^{6}$ P. Plotka, J. Nishizawa, T. Kurabayashi, and H. Makabe, IEEE Trans. Electron Devices 50, 867 (2003).

${ }^{7}$ Z. S. Gribnikov, N. Z. Vagidov, V. V. Mitin, and G. I. Haddad, J. Appl. Phys. 93, 5435 (2003).

${ }^{8}$ Z. S. Gribnikov, N. Z. Vagidov, V. V. Mitin, and G. I. Haddad, Physica E (Amsterdam) 19, 89 (2003).

${ }^{9}$ Z. S. Gribnikov, N. Z. Vagidov, and G. I. Haddad, J. Appl. Phys. 95, 1489 (2004).

${ }^{10}$ Handbook of Mathematical Functions, edited by M. Abramowitz and I. A. Stegun (Dover, New York, 1965). 